\title{
CORE POLARIZATION EFFECTS IN THE RELATIVISTIC QUANTUM-DEFECT-ORBITAL THEORY
}

\author{
D. BIELIŃSKA-WĄż $\dot{z}^{a}$, I. MARTin ${ }^{b}$ AND J. KARWOWSKI ${ }^{a}$ \\ a Institute of Physics, N. Copernicus University Grudziądzka 5, 87-100 Toruń, Poland \\ ${ }^{b}$ Departamento de Química Física, Facultad de Ciencias \\ Universidad de Valladolid, 47005 Valladolid, Spain
}

(Received December 1, 1993)

\begin{abstract}
The quantum-defect-orbital method has been reformulated in order to include both relativistic effects and the electron correlation described by a core polarization potential. All quantities appearing in this formulation may be evaluated analytically. A comparison with experimental results demonstrates, on one hand, significance of the relativity-correlation corrections and, on the other, inadequacy of the relativistic quantum-defect-orbital approach when indirect relativistic effects are important, i.e. when atoms contain closed shells of $d$ electrons.
\end{abstract}

PACS numbers: 32.70.Cs, 32.90.+a

\section{Introduction}

The quantum-defect-orbital (QDO) method has been formulated by Simons and Martin [1]. The method provides analytical atomic valence, Rydberg, and continuum orbitals from spectral data. The orbitals are exact eigenfunctions of an eigenvalue equation of a model Hamiltonian. Recently the same method has been derived using a supersymmetry-based approach [2]. A relativistic generalization of the QDO method has also been given [3].

The quantum defect orbitals (QDOs) may be expressed in terms of the confluent hypergeometric (Kummer) function. Therefore expectation values of many operators and transition integrals involving the dipole or velocity transition operators may be expressed analytically in both relativistic and nonrelativistic formulations of the method. However, introducing a correction which accounts for polarization of the atomic core leads to transition integrals which cannot be expressed analytically [4]. The core polarization effects are in some cases quite significant. Also the analytic nature of the method is its important advantage. In the present paper an approach which includes the effects of core polarization in 
a way which allows one to express all the quantities analytically has been developed. The approach has been applied to both nonrelativistic and relativistic QDO method and illustrated on several examples. A comparison with experimental data demonstrates significance of simultaneous inclusion of the relativistic and core polarization effects.

Another goal of the present study is to check to what an extent the relativistic QDO method may adequately describe relativistic effects in atoms containing closed shells of $d$ electrons. As it is well known [5], relativistic corrections to the energies and wave functions of the external electrons in atoms containing less than about 30 electrons are mainly due to their own motion (direct relativistic effects). In the case of atoms containing closed shells of $d$ and $f$ electrons, the relativistic effects influence the external electrons also through the relativistic rearrangement of the atomic core (indirect relativistic effects). The indirect effects are dominant in atoms of low ionization degree (particularly in the neutral ones). The relativistic QDO method, in its present formulation, is capable to describe the direct relativistic effects only. Therefore the accuracy of the results should deteriorate when the indirect effects become important. This expectation has been confirmed by calculations performed for neutral $\mathrm{Rb}$ and $\mathrm{Cs}$ atoms.

\section{The method}

The quantum defect orbitals $\Psi^{\mathrm{QD}}$ are the square-integrable solutions of a radial equation of the hydrogenic type

$$
\left[\frac{-\mathrm{d}^{2}}{\mathrm{~d} r^{2}}+\frac{\Lambda(\Lambda+1)}{r^{2}}-\frac{2 \zeta}{r}\right] \Psi^{\mathrm{QD}}(r)=2 \epsilon \Psi^{\mathrm{QD}}(r),
$$

where $\zeta, \Lambda$, and $\epsilon$ are constants determined by the quantum numbers $n$ and $\ell$, $k=j \pm 1 / 2$, the nuclear charge $Z$, the number of electrons $N$, and the experimental energy $E(n, \ell)$ or $E(n, \ell, j)$, depending on whether the fine structure splitting is taken into account or not. The nonrelativistic QDO equation differs from the relativistic one by the choice of the constants only [3].

In the nonrelativistic approach

$$
\begin{aligned}
& \zeta=Z-N+1, \\
& \Lambda=\ell+c-\delta, \\
& \epsilon=E(n, \ell) .
\end{aligned}
$$

The quantum defect $\delta$ is obtained from the following equation:

$$
E(n, \ell)=-\frac{\zeta^{2}}{2(n-\delta)^{2}}
$$

and $c$ is an integer constant chosen so that the radial functions are square integrable and possess the correct number of nodes.

In the relativistic approach

$$
\begin{aligned}
& \zeta=(Z-N+1)\left[1+\alpha^{2} E(n, \ell, j)\right], \\
& \Lambda=\tilde{n}-n+\ell+c-\delta, \\
& \epsilon=E(n, \ell, j)\left[1+\alpha^{2} E(n, \ell, j) / 2\right],
\end{aligned}
$$


where $\alpha$ is the fine structure constant and

$$
\tilde{n}=n-|k|+\sqrt{k^{2}-\alpha^{2} Z^{2}}
$$

is the "relativistic principal quantum number". The quantum defect is derived from

$$
E(n, \ell, j)=-\frac{\zeta^{2}}{2(\tilde{n}-\delta)^{2}}
$$

and $c$ is determined in the same way as in the nonrelativistic case.

The normalized solutions of Eq. (1) may be expressed in terms of the confluent hypergeometric (Kummer) functions

$$
F(a, b, z)=\sum_{m=0}^{-a} \frac{\langle a\rangle_{m}}{m !\langle b\rangle_{m}} z^{m},
$$

where

$$
\langle x\rangle_{m}=x(x+1)(x+2) \cdots(x+m-1) ; \quad\langle x\rangle_{0}=1
$$

is the Pochhammer symbol. The explicit expression is given by the following equation:

$$
\Psi^{\mathrm{QD}}(r)=\mathcal{N}(\nu r)^{\Lambda+1} \exp (-\nu r / 2) F(-K, 2 \Lambda+2, \nu r),
$$

where

$$
\begin{aligned}
& K=n-\ell-c, \\
& \nu=\frac{2 \zeta}{\Lambda+n-\ell-c}
\end{aligned}
$$

and

$$
\mathcal{N}=\left[\frac{\nu \Gamma(2 \Lambda+n-\ell-c+1)}{2(\Lambda+n-\ell-c) \Gamma(n-\ell-c)}\right]^{1 / 2} \Gamma(2 \Lambda+2)^{-1}
$$

is the normalization factor.

Transition probabilities between two states $|i\rangle$ and $|f\rangle$, where $i$ and $f$ stand for the appropriate sets of the relevant quantum numbers, are determined by the transition moment integrals

$$
R_{i f}=\int_{0}^{\infty} \Psi_{i}^{\mathrm{QD}}(r) Q(r) \Psi_{f}^{\mathrm{QD}}(r) \mathrm{d} r .
$$

If the transition operator $Q(r)$ corresponds to a multipole transition, it is expressed in terms of powers of the electron coordinates. If the velocity- or acceleration-like forms of the operator are used, then derivatives with respect to the electron coordinates appear. In all these cases the transition moment integrals may be expressed analytically. Introducing to the theory corrections which take into account the polarization of the atomic core by the optical electron, results in more complicated forms of the transition operator. For example, the dipole transition operator becomes $[4,6]$

$$
Q(r)=r\left[1-\frac{\alpha}{r^{3}} \eta\left(r, r_{\mathrm{c}}\right)\right]
$$


where $\alpha$ is the dipole polarizability of the core and $\eta\left(r, r_{\mathrm{c}}\right)$ is a cut-off function with $r_{\mathrm{c}}$ being a cut-off radius determined empirically. Recently the cut-off function originally proposed by Norcross [7]

$$
\eta\left(r, r_{\mathrm{c}}\right)_{1}=1-\exp \left[-\left(r / r_{\mathrm{c}}\right)^{3}\right]
$$

has been successfully used within the QDO approach [4]. However the resulting integrals are not expressible analytically. Therefore we selected another form of this function [8]:

$$
\eta\left(r, r_{\mathrm{c}}\right)_{2}=\left[1-\exp \left(-r / r_{\mathrm{c}}\right)\right]^{3} .
$$

Then, the resulting transition operator, for both $r \rightarrow 0$ and $r \rightarrow \infty$, retains the same asymptotic properties as $\eta\left(r, r_{\mathrm{c}}\right)_{1}$, but the transition integral $R_{i f}$ remains analytical.

\section{Results and discussion}

The oscillator strengths have been calculated for the sodium isoelectronic series, and for potassium, rubidium and cesium atoms. Only those cases for which experimental data are available have been considered. The results derived from the nonrelativistic/relativistic formulation are identified as QDO/RQDO. Three different forms of the transition operator (18) have been used: the one with $\eta=0$ (no core polarization effects), the one with $\eta=\eta_{1}$ (numerical integration in the transition moment integral is necessary), and the one with $\eta=\eta_{2}$ (analytical expressions for the transition moments). The resulting oscillator strength values have been identified as QDO0/RQDO0, QDO1/RQDO1 and QDO2/RQDO2, respectively.

The dipole polarizability of the core for $Z-N \geq 5$ have been calculated using the Thomas-Fermi model [9]. This approach is valid for highly ionized atoms only. Therefore for $Z-N<5$ the values of $\alpha$ have been taken from the Hartree-Fock tabulation by Fraga et al. [10].

The cut-off radius is usually selected in an empirical way. In Ref. [11] it was taken as twice the expectation value of $r$ calculated for the outermost orbital. In Ref. [4] it has been optimized for each atom. Other authors took different values for different transitions [12]. The dependence of the oscillator strengths of $4 s \rightarrow 5 p$ transitions in potassium on $r_{\mathrm{c}}$ is shown in Fig. 1. Results obtained using two different cut-off functions are here compared. Both curves are nearly parallel. Therefore one can get the same effect with any of the two functions taking different values of $r_{c}$. The oscillator strength values for $5 s \rightarrow n p(n=6,7)$, transitions in rubidium are plotted versus $r_{\mathrm{c}}$ in Fig. 2. The cut-off function $\eta_{2}$ has been used. As one can see, the results rather strongly depend on $r_{c}$. The optimum value of $r_{\mathrm{c}}$ may be correlated with the atomic core radius defined as either the expectation value of $\boldsymbol{r}$ for the outermost core orbital or as the location of the last maximum of this orbital. In order to retain simplicity of the method and to avoid its excessive flexibility by introducing arbitrary parameters, we have taken $r_{\mathrm{c}}=2 r_{\text {core }}$. The radius of the atomic core, $r_{\text {core }}$, according to Fraga et al. [10], has been defined as the position of the last maximum of the Hartree-Fock core 


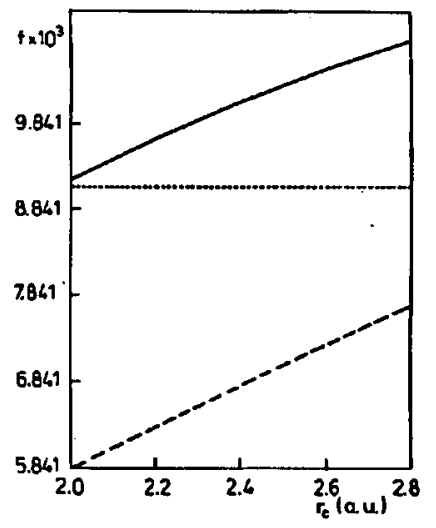

Fig. 1. RQDO oscillator strengths $f$ for $4 s \rightarrow 5 p$ transitions in potassium calculated with cut-off functions $\eta_{1}$ (broken line) and $\eta_{2}$ (solid line) plotted versus $r_{c}$. The dotted line corresponds to the experimental value.
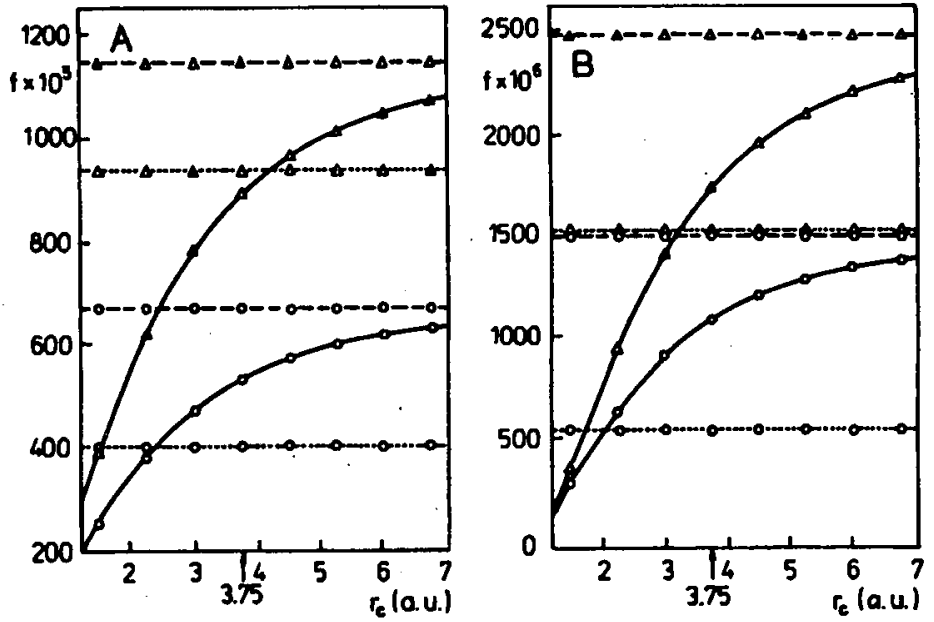

Fig. 2. Oscillator strengths $f$ for $5 s \rightarrow 6 p(\mathrm{~A})$ and $5 s \rightarrow 7 p(\mathrm{~B})$ transitions in rubidium plotted versus $r_{c}$. Symbols $O$ and $\Delta$ represent, respectively, transitions to ${ }^{2} P_{1 / 2}$ and to ${ }^{2} P_{3 / 2}$. In each case the results obtained with the core polarization neglected (broken line) and the core polarization included using the cut-off function $\eta_{2}$ (solid line) are compared with the experimental value (dotted line).

orbitals. The corresponding values of the cut-off radii, indicated in the figures, reasonably well correlate with the optimum empirical values.

In Table I oscillator strengths for several transitions in sodium isoelectronic series atoms are displayed. In Table II the oscillator strengths for some transitions in potassium are shown. As one can see, introducing the core polarization operator leads, in general, to better agreement with experiment. Also differences between 
TABLE I

Oscillator strengths for the transitions from $3 p{ }^{2} P_{3 / 2}$ to $3 d^{2} D_{5 / 2}$ (first entry), from $3 p^{2} P_{3 / 2}$ to $3 d^{2} D_{3 / 2}$ (second entry) and from $3 p{ }^{2} P_{1 / 2}$ to $3 d^{2} D_{3 / 2}$ (third entry) in sodium isoelectronic series atoms. All quantities are multiplied by $10^{3}$.

\begin{tabular}{c|r|r|r|r|r|r|r|r}
\hline \hline$Z$ & QDO0 & RQDO0 & QDO1 & RQDO1 & QDO2 & RQDO2 & MP $^{a}$ & \multicolumn{1}{c}{ CC $^{b}$} \\
\hline 15 & 714 & 713 & 697 & 695 & 703 & 702 & 642 & $620[14]$ \\
& 79 & 79 & 77 & 77 & 78 & 78 & 71 & $69[14]$ \\
& 793 & 786 & 774 & 776 & 781 & 784 & 716 & $700[14]$ \\
16 & 626 & 624 & 607 & 606 & 615 & 614 & 568 & $550[14]$ \\
& 70 & 69 & 67 & 67 & 68 & 68 & 63 & $62[14]$ \\
& 695 & 698 & 675 & 678 & 683 & 686 & 635 & $620[14]$ \\
20 & 407 & 405 & 390 & 388 & 398 & 396 & 372 & $376[14]$ \\
& 45 & 45 & 43 & 43 & 44 & 44 & 41 & $42[14]$ \\
& 453 & 458 & 433 & 438 & 443 & 448 & 421 & $424[14]$ \\
22 & 345 & 342 & 329 & 326 & 337 & 334 & 317 & $330[15]$ \\
& 38 & 38 & 37 & 36 & 37 & 37 & 35 & $36[15]$ \\
& 383 & 389 & 366 & 372 & 374 & 381 & 360 & $376[15]$ \\
26 & 264 & 260 & 251 & 247 & 258 & 254 & 243 & $253[15]$ \\
& 29 & 29 & 28 & 27 & 29 & 28 & 27 & $28[15]$ \\
& 293 & 302 & 279 & 287 & 286 & 295 & 282 & $294[15]$ \\
28 & 237 & 232 & 225 & 221 & 231 & 227 & 218 & $225[15]$ \\
& 26 & 25 & 25 & 24 & 26 & 25 & 24 & $25[15]$ \\
& 263 & 273 & 250 & 260 & 257 & 267 & 257 & $266[15]$ \\
\hline
\end{tabular}

${ }^{a}$ Model potential [13].

${ }^{b}$ Critical compilation.

TABLE II

Oscillator strengths for the transitions from $4 s{ }^{2} S$ to $n p{ }^{2} P(n=$ $5,6,7)$ in potassium. All quantities are multiplied by $10^{3}$.

\begin{tabular}{l|r|r|r|r|r|r|r}
\hline \hline & QDO0 & RQDO0 & QDO1 & RQDO1 & QDO2 & RQDO2 & CC $^{a}$ \\
\hline $4 s-5 p$ & 13.763 & 13.764 & 7.259 & 7.259 & 10.485 & 10.486 & 9.1 \\
$4 s-6 p$ & 2.684 & 2.682 & 0.906 & 0.907 & 1.767 & 1.768 & 0.9 \\
$4 s-7 p$ & 1.002 & 1.002 & 0.249 & 0.249 & 0.606 & 0.607 & 0.214 \\
\hline
\end{tabular}

${ }^{a}$ Critical compilation [14].

RQDO1 and RQDO2 oscillator strengths are not very significant. Therefore we conclude that using the cut-off function, which allows for analytical integration (Eq. (20)), is more appropriate.

The influence of the core polarization on the oscillator strengths along an isoelectronic series in RQDO and QDO approaches is illustrated in Fig. 3. Transitions in the sodium isoelectronic series atoms have been taken in this example. 
TABLE III

Oscillator strenghts for the transitions from the ground states $\left({ }^{2} S_{1 / 2}\right)$ of $\mathrm{Cs}$ and $\mathrm{Rb}$ atoms to $n p^{2} P_{1 / 2}$ (first entry) and $n p{ }^{2} P_{3 / 2}$ (second entry). The cut-off radii are chosen to be 3.75 a.u. for $R b$ and 4.75 a.u. for Cs.

\begin{tabular}{l|r|r|r|r|r|r|r}
\hline \hline \multicolumn{1}{l|}{} & QDO0 & RQDO0 & QDO2 & RQDO2 & Experiment & $e^{a}$ \\
\hline Rb & $5 p$ & 358 & 363 & 338 & 343 & $335[16]$ & 3 \\
& & 717 & 712 & 676 & 671 & $675[16]$ & \\
& $6 p$ & 604 & 668 & 473 & 530 & $400[17]$ & 5 \\
& & 1207 & 1145 & 947 & 892 & $937[17]$ & \\
& $7 p$ & 1324 & 1506 & 941 & 1093 & $560[17]$ & 6 \\
& & 2649 & 2476 & 1883 & 1506 & $1530[17]$ & \\
\hline Cs & $6 p$ & 363 & 377 & 339 & 352 & $330[18]$ & 3 \\
& & 727 & 713 & 678 & 665 & $660[18]$ & \\
& \multirow{2}{*}{$7 p$} & 586 & 751 & 441 & 584 & $277[19]$ & 5 \\
& & 1171 & 1021 & 883 & 755 & $1200[19]$ & \\
\hline
\end{tabular}

a The oscillator strengths for the same transition have been multiplied by the power of 10 given in the corresponding entry.

However the general behaviour in other cases is similar:

- the core polarization effects reduce the oscillator strength values;

- for large values of $Z$ the core polarization and the relativistic effects are non-additive.
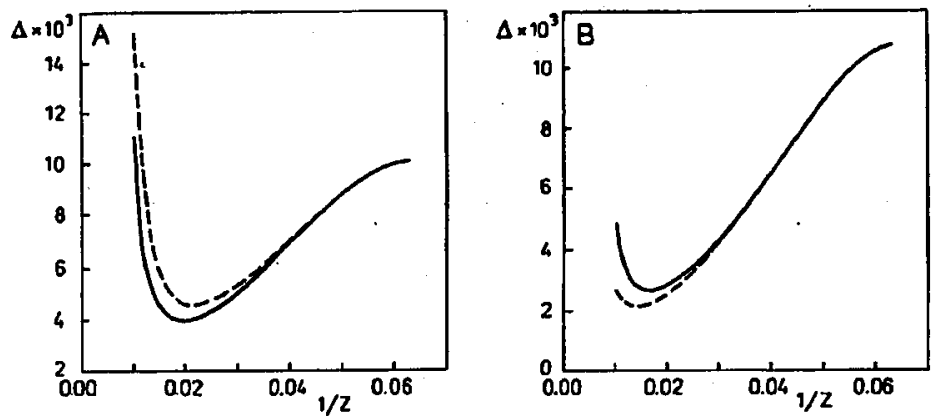

Fig. 3. The influence of the core polarization and the relativistic effects on the oscillator strengths of $3 s^{2} S_{1 / 2} \rightarrow 3 p^{2} P_{3 / 2}$ (A) and $3 p^{2} P_{3 / 2} \rightarrow 3 d^{2} D_{5 / 2}$ (B) transitions in sodium isoelectronic series atoms. The curves represent differences $\Delta$ between the oscillator strength values calculated with core polarization effects neglected and included. The solid lines correspond to the relativistic and the broken ones to the nonrelativistic values. 
The oscillator strengths for $n s \rightarrow n^{\prime} p$ transitions in $\mathrm{Rb}$ and Cs atoms are shown in Table III. As one should expect, the core polarization effects are here very large (cf. Figs. 1 and 2). However relativistic effects are described incorrectly in these cases. Only the very small direct relativistic effects resulting from the interaction between the singly charged atomic core and the optical electron are taken into account. Therefore the calculated intensity ratios of ${ }^{2} S_{1 / 2} \rightarrow{ }^{2} P_{3 / 2}$ and ${ }^{2} S_{1 / 2} \rightarrow{ }^{2} P_{1 / 2}$ transitions remain close to 2 , while the experimental ones range from 2 (as in $5 s \rightarrow 5 p$ transition in $\mathrm{Rb}$ ) to 4 (as in $6 s \rightarrow 7 p$ transition in Cs). This inadequacy of the description shows limits of applicability of the QDO theory. These limits may be extended only by allowing the indirect relativistic effects to influence the wave function of the optical electron.

\section{References}

[1] G. Simons, J. Chem. Phys. 60, 645 (1974); I. Martin, G. Simons, J. Chem. Phys. 62, 4799 (1975).

[2] V.A. Kostelecky, M.M. Nieto, Phys. Rev. A 32, 3243 (1985).

[3] I. Martin, J. Karwowski, J. Phys. B, At. Mol. Opt. Phys. 24, 1539 (1991); J. Karwowski, I. Martin, Phys. Rev. A 43, 4832 (1991).

[4] I. Martin, C. Barrientos, Can. J. Phys. 64, 867 (1985); C. Barrientos, I. Martin, J. Mazon, J. Chem. Phys. 84, 715 (1987).

[5] I.P. Grant, Int. J. Quantum Chem. 25, 23 (1984).

[6] S. Haamed, A. Herzenberg, M.G. James, J. Phys. B, At. Mol. Phys. 1, 822 (1968).

[7] D.W. Norcross, Phys. Rev. A 7, 606 (1973).

[8] D. Bielińska-Waqz, MSc Thesis, Nicholas Copernicus University (1992), in Polish.

[9] V.P. Shevelko, A.V. Vinogradov, Phys. Scripta 19, 275 (1979); B.N. Chichkov, V.P. Shevelko, Phys. Scripta 23, 1055 (1981).

[10] S. Fraga, J. Karwowski, K.M.S. Saxena, Handbook of Atomic Data, Elsevier, Amsterdam 1976.

[11] M. Szulkin, J. Karwowski, J. Phys. B, Al. Mol. Phys. 14, 4729 (1981).

[12] J.W. Weisheit, Phys. Rev. A 5, 1621 (1972); D. Ilofsaess, Z. Phys. A 281, 1 (1977).

[13] C.E. Theodosiu, L.J. Curtis, Phys. Rev. A 38, 4435 (1988).

[14] W.L. Wiese, M.W. Smith, B.M. Miles, Atomic Transition Probabilities, Vol. 2: Sodium through Calcium, National Stand. Ref. Data Ser., Nat. Bur. Stand. (U.S.), Washington DC 1969.

[15] G.A. Martin, J.R. Fuhr, W.L. Wiese, J. Phys. Chem. Ref. Data 17, Supp. 3 (1988).

[16] J.K. Link, J. Opt. Soc. Am. 56, 1195 (1966).

[17] E. Caliebe, K. Niemax, J. Phys. B, At. Mol. Phys. 12, L45 (1979).

[18] W. Happer, Rev. Mod. Phys. 44, 169 (1972).

[19] R.J. Exton, J. Quant. Spectrosc. Radiat. Transfer 16, 309 (1976). 